\title{
ESTIMACIÓN DE UNA FUNCIÓN DE COSTOS DE PRODUCCIÓN EN EL CULTIVO DEL ALGODÓN EN EL VALLE DEL SINÚ
}

\section{ESTIMATION OF A FUNCTION OF PRODUCTION COSTS IN THE COTTON CROP IN THE VALLEY OF SINU}

\author{
Antonio M. Martínez
}

Recibido para evaluación: Junio 27 de 2010 - Aceptado para publicación: Noviembre 30 de 2010

\begin{abstract}
RESUMEN
El objetivo principal del trabajo fue analizar la racionalidad del productor algodonero referente al uso de insumos que constituyen la estructura de costos con el fin de hacer recomendaciones que permitan mejorar la competitividad del cultivo. Se presenta una evidencia de la aplicación de la teoría neoclásica en el caso del cultivo de algodón en el Valle del Sinú, departamento de Córdoba en Colombia. Los resultados están sustentados en datos del Plan de Algodón de CORPOICA a través de 108 encuestas tomadas en campo en el año 2005. Los datos fueron ajustados según el Índice de Precios al Productor del Banco de la República para ser actualizados a precios de 2009. Los supuestos empleados son el de la minimización de los costos y la racionalidad del productor. Se formuló el modelo teórico y el modelo matemático, se hizo la estimación de los parámetros y se procedió al análisis de la información y discusión de resultados, llegando a determinar un costo mínimo. Este trabajo permitió evidenciar la validez de la teoría neoclásica de la función de producción aplicada al caso específico del cultivo del algodón y la asignación irracional de los recursos al momento de utilizar factores de producción.
\end{abstract}

Palabras clave: Costo, producción, racionalidad, eficiencia.

\begin{abstract}
This paper aims at analyzing the rationality of cotton producer regarding the use of inputs that represent the costs structure in order to make recommendations to improve crop competitiveness. We present evidence for the application of neoclassical theory in the case of cotton cultivation in the Valle del Sinú, department of Cordoba in Colombia. The results are supported by field work data supplied by the CORPOICA Cotton Plan through 108 field surveys carried out in 2005. The data were adjusted according to Colombian Bank of the Republic Producer Price Index, to be updated to 2009 prices. The assumptions used are the minimization
\end{abstract}

Economista Ph.D. Corporación Colombiana de Investigación Agropecuaria CORPOICA. Centro de Investigación Tibaitatá, Cundinamarca - Colombia. Email: antoniomarti40@hotmail.com 
of costs and producer rationality. Theoretical and mathematical models was formulated, the estimation of parameters was made, then the analysis of information and discussion of results, leading to determining a minimum cost. Finally, this work allows conclude the validity of the neoclassical theory of production function applied to the specific case of cotton cultivation and irrational allocation of resources at the time of use factors of production.

Key words: Cost, production, rationality, efficiency

\section{INTRODUCCIÓN}

El Algodón es la fibra natural más usada en la industria mundial de textiles, cuya producción mundial se estima en 24 millones de toneladas de fibra por año, en 130 países y ocupa el $2,5 \%$ de la superficie agrícola del planeta, con una superficie de cultivo de 34 millones de hectáreas. Colombia participa con el $0,14 \%$ de la producción mundial, con cerca de 35.000 toneladas de fibra año-1 ${ }^{-1}$ No obstante, ocupa el puesto 14 por productividad, con un rendimiento promedio de 775 kilogramos de fibra $\mathrm{ha}^{-1}$, registrando un incremento del 4,1\% sobre el promedio mundial (Comité Consultivo Internacional del Algodón-ICAC 2009).

Los costos de producción en el año 2010 ascendieron a 5.144.299 pesos ha-, donde se destaca como el componente de mayor participación los agroquímicos con una participación del $28 \%$ sobre el total de costos de producción y un ingreso neto después de deducidos los costos de 1.002.265 pesos ha-, con una rentabilidad del 19,8\%. Cabe destacar que el gobierno Colombiano cada año fija un precio mínimo de referencia con el fin de proteger la producción nacional, por considerar que Colombia es deficitaria y además, el cultivo revista importancia por cuanto genera unos 19.297 empleos directos por campaña, representando el 4,5\% de los empleos directos registrados en los cultivos transitorios (Agrocadenas 2010 ) .

El trabajo de tipo empírico se basa en la teoría neoclásica de la función de producción, se demostró su aplicación a un caso práctico como es el cultivo del algodonero en el Valle del Sinú, departamento de Córdoba, Colombia; los datos provienen de 108 unidades productivas obtenidos mediante una encuesta aplicada por el componente de transferencia del Plan de Algodón liderado por CORPOICA en el año 2005. Los elementos constitutivos del costo fueron: los costos indirectos especialmente administración, intereses del capital, alquileres; los costos directos los constituyen los agroquímicos, la mano de obra, maquinaria y servicios, los cuales sumados los dos componentes dan el costo total de producción para una hectárea de algodón.

El uso del método matemático y en especial la estimación de funciones de costos se han usado en muchos estudios económicos en todos los sectores de la actividad económica que conducen al estudio de la eficiencia del uso de factores de producción. En Agricultura en el período comprendido entre 1993 y 1999 de acuerdo con el catálogo de AGRIS, se encontraron más de 160 trabajos publicados en revista norteamericanas, europeas, australianas 
en cultivos como arroz, algodón, yuca, maíz, trigo y papa (FAO 2010).

La literatura sobre aplicación de la función de producción en el caso de la producción agrícola tiene sus orígenes en los trabajos de Tintner (1944), el cual fue seguido por Heady (1946). Un análisis de la evolución de la función de producción en estudios empíricos se puede encontrar en Mundlak (2001), comentado en detalle por Herford en su libro "La competitividad de la agricultura en las Américas". Para el caso colombiano se encuentran los trabajos de Flórez et al. (1978), quienes estimaron una función de respuesta para el trigo en la sabana de Bogotá a la aplicación de fertilizantes con dosis diferentes de nitrógeno y fósforo. Posada (1984) estimó una función de respuesta de la caña de azúcar a la fertilización con nitrógeno, fósforo y potasio; Castillo et al. (2003) estimaron una función de producción para el caso del maíz amarillo en el Valle del Sinú, donde muestran la inexistencia de economías de escala y los diferentes niveles de eficiencia de acuerdo con el sistema de producción. Por su parte, Martínez (2002) estimó una función de costos para el secado de yuca, la cual indica que existe subutilización del factor capital principalmente.

Con relación a las aplicación de funciones de costos se busca demostrar si existe racionalidad en la asignación de los recursos en la medida que aumente la escala de producción, generando economías de escala. En el caso de la agricultura se esperaría que cuando la producción se expande, el costo por unidad de producción tienda a reducirse, ya sea porque hay una distribución de los costos fijos. El término economías de tamaño es usado para describir una situación en la que cuando se aumenta la cantidad de producto se va disminuyendo el costo fijo promedio (economías de tamaño no pecuniarias), o porque algunos costos variables se reduzcan por compras en grandes proporciones de insumos (Debertin 1989).

De cualquier forma el aumento de la eficiencia se puede dar si se racionaliza el uso de los insumos mediante la disminución del costo unitario bajo el supuesto de que los precios no bajen considerablemente, esto es lo que se propone demostrar con este trabajo. La hipótesis que subyace de esta investigación es que pese a que el agricultor se preocupa por trabajar con un costo bajo termina por subutilizar recursos.

El objetivo de este trabajo fue analizar la racionalidad del productor algodonero referente al uso de insumos que constituyen la estructura de costos con el fin de hacer recomendaciones que permitan mejorar la competitividad del cultivo. El supuesto del cual se parte en esta investigación es el de la minimización de los costos y la racionalidad del productor con el objetivo de analizar la racionalidad del productor respecto a la utilización de factores de producción. Apoyados en este principio se formuló la función de costos de producción que muestra los componentes constitutivos y los respectivos aportes que cada elemento hace al costo total. La determinación de un costo mínimo está acompañada por un máximo en la función de producción generándose aumentos en la producción cada vez que se utilizan unidades adicionales de factores de producción o cada 
vez que se aumenta la escala de producción (Debertin 1989). Cabe anotar, que a pesar de que los datos de campo corresponden a la cosecha 2005 - 2006 con el uso del índice de precios al productor IPP para actualizar las datos a 2009, los costos totales por hectárea se estimaron en 5.144.299 pesos ha-1, un tanto superiores a los que registró CONALGODÓN para la cosecha 2008 - 2009, los cuales corresponden a 4.581 .165 pesos ha-1. Esto permite tener cierta seguridad con respecto a la actualidad y veracidad de los datos.

\section{MATERIALES Y MÉTODOS}

Para la construcción del modelo matemático, se utilizó la información generada por la Corporación Colombiana de Investigación Agropecuaria CORPOICA, obtenida mediante la aplicación de unas encuestas sobre costos de producción realizadas en 108 fincas productoras de algodón coordinadas por el ingeniero agrónomo Francisco Negrete Barón.
Con los formularios diligenciados se elaboró una base de datos, la cual sirvió para realizar las pruebas estadísticas y demás análisis realizados.

Los datos originales se organizaron en archivos de excel para proceder a hacer los cálculos y conversiones como actualización de los datos 2009 y la transformación en logaritmos para poder medir la elasticidad de los diferentes componentes del costo de producción o la respuesta de los diferentes componentes cuando aumentan las unidades producidas. Seguidamente se hizo la formulación y estimación del modelo. La base de datos de excel no se presenta en este trabajo, solo se hace un resumen en la tabla 1 con los promedios de las 108 fincas encuestadas.

La explicación de los costos de producción para la siembra de una hectárea de algodón semilla se basa en la teoría neoclásica de la función de producción tipo Cobb Douglas

Tabla 1. Resultados de la estimación del Modelo de la función de Costos de Producción de una hectárea de algodón en el Valle del Sinú 2009

\begin{tabular}{crrrr}
\hline \multicolumn{1}{c}{ Variable } & \multicolumn{1}{c}{ Coefciente } & \multicolumn{1}{c}{ Std. Error } & \multicolumn{1}{c}{ t-Static } & \multicolumn{1}{c}{ Prob } \\
\hline C & 0,926423 & 0,084485 & 10,96555 & 0,0000 \\
LOG(IND) & 0,557953 & 0,005882 & 94,86427 & 0,0000 \\
LOG(AGROQ) & 0,278550 & 0,004653 & 59,87072 & 0,0000 \\
LOG(MAQ) & 0,026626 & 0,002526 & 10,53987 & 0,0000 \\
LOG(MDO) & 0,1201954 & 0,0047682 & 25,21 & 0,0000 \\
LOG(SER) & 0,032829 & 0,003313 & 9,907932 & 0,0000 \\
$R^{2} \quad 0,996826$ & & & \\
$R^{2}$ ajustado & 0,996671 & & & \\
S.E. de regresión & 0,011157 & & & \\
Suma de cuadrados & 0,012697 & Schwarz criterion & & \\
Durbin-Watson stat & 2,0 & 0,000000 & & \\
\multicolumn{2}{c}{ 2,020247 } & Prob(F-statistic) & 0,050510 & \\
\hline
\end{tabular}

Fuente: Elaboración propia con base en la encuesta de CORPOICA Plan de Algodón 2009. 
que relaciona la cantidad de insumos que se utilizan en la producción y permite calcular las cantidades máximas de producto que se pueden obtener con una cantidad dada de insumos. La función Cobb-Douglas es una forma de función de producción, ampliamente usada para representar las relaciones entre un producto y las variaciones de los insumos tecnología, trabajo y capital. Fue propuesta por Knut Wicksell (1924) e investigada con respecto a la evidencia estadística concreta por Charles Cobb y Paul Douglas en 1928.

$\mathrm{CT}=\mathrm{F}(\mathrm{K}, \mathrm{L})=\mathrm{AKaLb}$

Donde CT: es el Costo Total; $\mathrm{K}$ y $\mathrm{L}$ son los factores capital y trabajo, respectivamente; $\alpha$ y $\beta$ son parámetros que indican la elasticidad con respecto a los dos factores, es decir el cambio que experimenta el costo total cuando cambian las unidades de los factores capital y trabajo.

Apoyados en este principio se formuló la función de costos de producción que muestra los componentes constitutivos y los respectivos aportes que cada elemento hace al costo total.

Se parte del supuesto de la minimización de los costos y la racionalidad del productor. Con la información que se obtuvo de los datos de las encuestas en campo se formuló un modelo que presenta la función de costos para una hectárea cultivada de algodón.

Con el objeto de determinar la función de costos de producción de una hectárea de algodón se formuló el modelo (2)
$\mathrm{LOG}(\mathrm{CT})=\mathrm{C}(1)+\mathrm{C}(2) * \mathrm{LOG}(\mathrm{IND})+$ $\mathrm{C}(3) * \mathrm{LOG}(\mathrm{AGROQ})+\mathrm{C}(4) * \mathrm{LOG}(\mathrm{MDO})+$

$\mathrm{C}(5) * \mathrm{LOG}(\mathrm{MAQ})+\mathrm{C}(6) * \mathrm{LOG}(\mathrm{SER})$

El Modelo General es

$Y=\beta_{0}+\beta_{1} X_{1}+\beta_{2} X_{2}+\beta_{3} X_{3}+\beta_{4} X_{4}+\beta_{5} X_{5}+e$

Donde:

$\mathrm{Y}=$ Costo Total

$\beta=$ Los parámetros o estimadores habrá tantos como variables explicitarías haya.

$\mathrm{X}=$ Las variables explicotearías

e = Término de perturbación.

LOGCOSTO TOTAL $=\mathrm{C} 0+\operatorname{COS} *($ IND $)+$ $\mathrm{COST}^{*}(\mathrm{AGROQ}) \mathrm{COS}^{*}(\mathrm{MDO})+\mathrm{COS}^{*}(\mathrm{MAQ})+$ COS* $($ SER $)+\mathrm{e}$

Costo Total: Indica el costo total de cultivar una hectárea de algodón semilla.

$\mathrm{C}_{0}=$ Intercepto no tiene significado económico.

\section{Costos Indirectos}

$\mathrm{C}(2)$ COS IND $=$ Costos indirectos especialmente administración, alquileres.

\section{Costos Directos}

C(3) AGROQ = Costos por concepto control de plagas y enfermedades.

C(4) COS MDO = Costos de la mano de obra incluye jornales y personal permanente.

C(5) MAQU = Costos por concepto de Maquinaria y equipos. 
C(6) COS SER = Costos por servicios prestados como bodegas, transporte, fletes.

La estimación del modelo se hizo utilizando el paquete econométrico Evieweus versión 5.0, por tanto la salida que se presenta es la de este programa. El método de estimación utilizado fue el de los Mínimos Cuadrados Ordinarios con un coeficiente de determinación de 0,99 y alta significancia, lo que quiere decir que las variables utilizadas para explicar el costo son suficientes y en estas circunstancias, el modelo es consistente. Los coeficientes reúnen las características de insesgo, varianza mínima y homosedasticidad, aptos para garantizar una buena estimación del modelo como el Durbin Wason de 2.0 lo que indica no correlación serial y por tanto la robustez del modelo.

\section{RESULTADOS Y DISCUSIÓN}

La salida del computador producto de la estimación se presenta en la tabla 1, la cual generó información consistente, dadas las características estadísticas de los estimadores como insesgo, homosedaticidad y no correlación serial de los parámetros.

La estimación de los datos por el método de los mínimos cuadrados presenta la función de costos a través de los coeficientes de las variables que generan la siguiente función:

LOG $(\mathrm{CT})=0,926+0,557 * \mathrm{LOG}(\mathrm{IND})+$ $0,278 *$ LOG(AGROQ) $+0,120 *$ LOG(MDO) $+0,0266 *$ LOG $(M A Q)+0,0328 *$ LOG(SER) (4)

Lo que muestra la estimación es que el 55\% de los costos totales está explicado por los costos indirectos, el $27 \%$ por los costos de insecticidas, fungicidas, fertilizantes y demás elementos provenientes de la industria química y biológica. El $12 \%$ de los costos lo explica la mano de obra; por su parte la maquinaria lo hace con un $2 \%$ y por último los servicios con un $2 \%$ de la explicación de los costos.

La función permite calcular las cantidades de cada uno de los componentes que se deben utilizar para maximizar los ingresos, seguidamente se procede a reemplazar la información del trabajo de campo en la ecuación obtenida por la regresión, para lo cual es necesario sustituir en la ecuación la demanda de cada uno de los componentes o factores de producción.

Se halla la función de costos sustituyendo en cada uno de los componentes así:

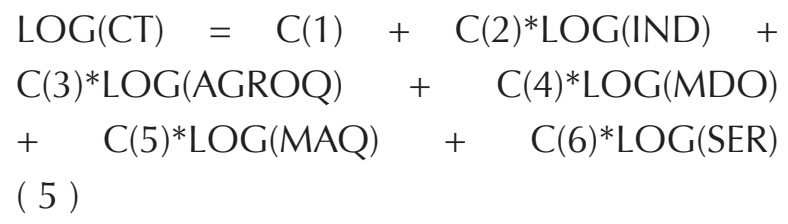

$\mathrm{CT}=0,9264230094+(2.872 .978,97)^{0.559409}+$ $(1.430 .809,34)^{278953}+579.044,73^{0.119987}+(112$. $068,9)^{.02662}+(149.397,5)^{0.033167}(6)$

$\mathrm{R} 2=0.9968826$

Los exponenciales corresponden a los coeficientes estimados en la regresión.

En este caso se presentan los valores que se colocan entre paréntesis $(2.872 .978,90)$ que corresponden al valor incurrido en una hectárea por concepto de costos indirectos, expresados en pesos colombianos, discriminados en agroquímicos $(1.430 .809,34)$, mano de obra 
(579.044,43), maquinaria agrícola $(112.068,9)$ y los servicios $(149.397,5)$ en que se incurre para sembrar una hectárea de algodón.

Remplazando con los precios de cada uno de los factores de producción se llega al costo mínimo de producción.

$\mathrm{CT}=2.125 .581,79$ pesos por hectárea. Este es el costo mínimo de producir una hectárea de algodón.

Es necesario comentar que de las 108 fincas que a las cuales se les aplicó la encuesta, el promedio de costo por hectárea fue de 5.144.299 pesos por hectárea, sin embargo, la función de costos aquí obtenida presenta un valor inferior, lo que significa que se está trabajando con un costo por encima del mínimo y en ultimas que se está subutilizando los factores tierra y capital representado en la infraestructura y adecuación del suelo, obras como riego, canales y maquinaria y especialmente el componente administrativo que tiene un alto impacto en el total de los costos en más del 50\% cuando se incremente una unidad producida.

Esta función permite simular los diferentes cambios que puedan presentarse en el uso de los insumos y en la producción para ver los cambios en el costo total.

La determinación de un costo mínimo está acompañada por un máximo en la función de producción generándose aumentos en la producción cada vez que se utilizan unidades adicionales de factores de producción o cada vez que se aumenta la escala de producción.

\section{CONCLUSIONES}

Se evidencia la validez de la teoría neoclásica en la construcción de una función de costos aplicada al caso específico del cultivo del algodonero en el Valle del Sinú, al igual que permite hacer inferencia sobre la racionalidad del productor.

El estudio muestra que a pesar de que el productor tiende a ser racional termina subutilizado factores de producción, en este caso el factor capital y trabajando con costos superiores en un 41 por ciento por encima del costo mínimo estimado en la función comprometiendo su eficiencia y competitividad.

Los resultados permiten inferir que los problemas de ineficiencia en el cultivo del algodonero están muy relacionados con la administración y la gestión, lo que quedó demostrado en los resultados de la regresión con un coeficiente del $55.7 \%$ que indica que es un componente muy importante en la estructura de costos de producción.

Es necesario realizar evaluaciones para conocer el grado de ineficiencia productiva para establecer el factor que se está subutilizando, lo cual conlleva a un análisis técnico económico para racionalizar el uso de los factores de producción para mejorar la eficiencia y aumentar la competitividad de la empresa algodonera. 


\section{REFERENCIAS}

Observatorio de competitividad Agrocademas.

2010. IICA. Http-/: www.agrocadenas .gov.co [enero de 2010].

Castillo 2003, Costos y margen bruto de ganancia en el cultivo del maíz amarillo en la subregión del Sinú Medio.

Cobb, C.W y Douglas P, 1928. A Theory of Production, American Economic Review 18 (supplement): 139-165 .

\section{Comité Consultivo Internacional del Algodón} 2009.

Derbertin, D. 1989. Agricultural production economics. Macmillan 151 - 153.

Florez, V, Acosta, J y Navas, J., 1978. Análisis agroeconómico de la fertilización en cultivos, Bogotá. Instituto Colombiano Agropecuario, 267 p (boletín técnico 46)

Heady (1946). Production Funtion and Suppy Diario de 28:987-1004 Economía agrícola.

Hertford R, García, J A, 1999. La Competitividad de la Agricultura en las Américas. Serie CIAT economía e impacto. Cali Colombia 240 p.
Martínez, A, 2002. Análisis de la Viabilidad Económica y Financiera de Sistemas de Secado Artificial de Yuca en los departamentos de Córdoba y Sucre. Tesis de Maestría Universidad Nacional de Colombia Bogotá 150 p.

Mundlak, Y, 1996. "Estimación de la función de producción: Reviviendo el Primal", ... Mundlak, Yair, 2001. "La producción y la oferta," Manual de Agricultura.

Nicholson Walter 1997. Teoría Microeconómica "Principios y Aplicaciones" Editorial McGraw Hill .

Posada, R (1984). "Análisis Económico de los experimentos con fertilizantes en Caña de Azúcar" En: Colombia. Memorias Primer congreso de la sociedad colombiana de técnicos de la caña de azúcar CENICAÑA.

Serpa, M 2004. Análisis económico del sistema de producción de maíz amarillo en el Sinú, Dpto de Córdoba. Tesis de Maestría. Universidad Nacional de Colombia Bogotá. 120p.

Timer, G. 1944. La función de producción en las granjas del estado de lowa .

Wicksell (1924). On the Origin of the CobbDouglas Production Function." Economy and History reprinted (1971) 